Revista Latinoamericana de la Papa 22 (1): 38 - 48

http://www.papaslatinas.org/revista.html

ISSN: $1853-4961$

\title{
Definición de repertorios de cultivares de papa para agricultores altoandinos en un contexto de cambio climático
}

\author{
X. Cadima ${ }^{1 / *}$, I. López ${ }^{2}$
}

Recibido: $13 / 04 / 2018$

Aceptado: 06/06/2018

Accesible en línea: Junio de 2018

\section{Resumen}

En Bolivia, en la región de los Andes la papa es el principal cultivo, en el que están involucrados cerca de 200.000 familias, que cultivan papa en más de 170.000 ha anualmente. La semilla utilizada para esta siembra proviene en un $97 \%$ del sistema tradicional, es decir de los pequeños agricultores de zonas altas, que proveen semilla no certificada. Aunque este sistema también maneja diversidad, su mayor debilidad es la disminución de la calidad de la semilla (tanto genética como sanitaria). Para mejorar los procesos de definición de cultivares para la producción de semilla, y asegurar así la disponibilidad de una mayor diversidad del cultivo en forma de semilla de calidad que podría complementar tanto a los sistemas formales como informales, se trabajó en el desarrollo de un enfoque de definición de repertorios de cultivares de papa en un contexto de cambio climático utilizando información de diferentes niveles y atributos de un set de 37 cultivares, los cuales fueron analizados mediante un Análisis de Factores para Datos Mixtos. El análisis permitió identificar, tendencias de grupos de cultivares que respondan a las necesidades y preferencias de los agricultores asociadas a condiciones medioambientales, preferencias agronómicas, de mercado y valores culturales.

Palabras claves adicionales: Pequeños agricultores, Semilla, Rasgos funcionales, Cambio climático.

\section{Performance of two potato varieties under water stress}

\section{Summary}

In Bolivia, in the Andes region, the potato is the main crop. Nearly 200.000 families grow potatoes in more than 170.000 ha annually. The seed used for planting comes in about $97 \%$ from the traditional system, that is, from small farmers in the highlands who provide non-certified seed. Although this system also manages diversity, its greatest weakness is a decrease in the quality of the seed (both genetic and phytosanitary). To improve the processes of definition of cultivars for seed production, and thus ensure the availability of greater diversity of the crop in terms of quality seed, to complement both formal and informal systems, we worked on developing a definition of portfolios of potato cultivars in a context of climate change using information from different levels and attributes in a set of 37 cultivars, which were analyzed through a Factor Analysis for Mixed Data. The analysis allowed identifying trends of groups of cultivars that respond to the needs and preferences of farmers associated with environmental conditions, agronomic preferences, market and cultural values.

Additional keywords: Small farmers, Seed, Functional traits, Climate change.

\footnotetext{
* Autor para correspondencia. Correo electrónico: x.cadima@proinpa.org

${ }^{1}$ Fundación PROINPA, Cochabamba, Bolivia.

${ }^{2}$ Bioversity International.
} 


\section{Introducción}

En Bolivia al menos el $49 \%$ de la población se autoidentifica con algún pueblo indígena originario campesino, la mayor parte son pequeños agricultores aymaras y quechuas de zonas altas del país que viven en el área rural practicando agricultura tradicional de bajos insumos siendo ésta la base de su economía y sustento familiar (Eyzaguirre 2015). En manos de estos pequeños agricultores también está el mantenimiento de la diversidad genética agrícola. Un componente fundamental del sistema de agricultura tradicional de altura en Bolivia es la papa (Solanum tuberosum), de la cual el país alberga una enorme diversidad: alrededor de 1000 cultivares nativos (Cadima y Gandarillas 2009).

La papa es el cuarto cultivo más producido en Bolivia, con más de 192000 has de papa cultivada en el país (INE 2018). La semilla requerida para esa superficie de cultivo proviene de dos sistemas: formal e informal o tradicional. El sistema formal, regulado por normas nacionales, provee menos del $5 \%$ de la semilla requerida, y solo dos cultivares de alto valor comercial representan el $95 \%$ de esa producción. El sistema tradicional manejado por agricultores, provee hasta el 97\% de la semilla de papa usada en el país (semilla no certificada) de un repertorio mayor de cultivares pero de forma informal y sin garantía de la calidad de la semilla (Escobar 2014).

Existe una enorme diversidad de papa en Bolivia pero el mercado de semillas (tanto formal como informal) aprovecha una parte muy limitada de esta diversidad. Existe por lo tanto un gran potencial para diversificar la semilla de papa, identificando y multiplicando aquellos cultivares que son los más apropiados para diferentes situaciones ambientales y socio-económicas. Con este objetivo, PROINPA desarrolló en Bolivia un proyecto llamado "Mejora de los Sistemas de Semillas para la Seguridad Alimentaria de los Pequeños Agricultores", liderado a nivel global por Bioversity International y financiado por la COSUDE. En este documento se muestra un enfoque trabajado en el proyecto para identificar un repertorio de cultivares, que busca las complementariedades entre cultivares más que similitudes. El enfoque es aplicado para identificar diferentes niveles y atributos de cultivares de papa ante un contexto de cambio climático percibido por los productores de papa en un centro de alta agrobiodiversidad en Bolivia.

\section{Materiales y Métodos}

Características del Área de Trabajo. El municipio de Colomi en Cochabamba-Bolivia fue elegido como el área de colección de datos. Este municipio es considerado un centro de alta agrobiodiversidad por sus características climáticas y ecológicas que han dado lugar a la diversificación de cultivos y variabilidad intraespecífica (Cadima et al. 2016, García y Cadima 2003). Se eligió este lugar porque es un municipio agrícola en el que la agrobiodiversidad es la base de la seguridad alimentaria y económica de sus habitantes, y está expuesto como otras regiones andinas, a estreses climáticos. En este municipio se distinguen dos pisos ecológicos: 1) Puna, con un rango altitudinal de 3.000 y $4.800 \mathrm{~m}$ de altitud, clima frío húmedo invernal y frío húmedo a subhúmedo, temperatura promedio de $15^{\circ} \mathrm{C}$, precipitación anual de $800 \mathrm{~mm}$ y humedad relativa del $55 \%$. Los tubérculos andinos (papa, oca, papalisa e isaño) son los más representativos de la diversidad agrícola, y 2) Trópico, con un rango altitudinal de 100 y $3.000 \mathrm{~m}$ de altitud, clima frígido de puna y tórrido de los llanos, humedad relativa media del $75 \%$, precipitación pluvial de 2.000 a $3.500 \mathrm{~mm}$ anuales y temperatura media de $20^{\circ} \mathrm{C}$. La diversidad agrícola en el trópico de Colomi es abundante en hortalizas locales e introducidas, frutales, leguminosas y raíces andinas (GAM Colomi 2008).

Diversidad de papa de Colomi. Para la presente investigación se tomaron en cuenta 37 cultivares de papa sujetos a evaluación por los agricultores de Colomi en el periodo 2011 a 2015. Estos 37 cultivares incluían 30 cultivares nativos de las especies $S$. 
juzepczukii, (3x), S. goniocalyx (2x), $S$. stenotomum (2x), S. phureja (2x) y $S$. tuberosum subsp. andigena (4x) y siete cultivares mejorados ( $S$. tuberosum). Todos los cultivares fueron evaluados bajo los mismos parámetros, no se hizo un muestreo por atributo o rasgo funcional, tampoco se hizo una diferenciación por su condición de nativos o mejorados. La evaluación consideró el material genético disponible en los campos de los agricultores.

Percepción local de las principales limitaciones en la producción de papa en el Municipio de Colomi por efecto del cambio en el clima. Los eventos climáticos como variaciones en las lluvias, granizadas y heladas han sido siempre limitaciones en la puna altoandina, sin embargo los pobladores locales mencionan que el clima ha empeorado los últimos 20 a 30 años, identificando como principales problemas abióticos: heladas y granizadas impredecibles, periodos largos de sequía, lluvias intensas irregulares provocando saturación de la humedad del suelo en zonas bajas. Además problemas bióticos, plagas y enfermedades, cuya incidencia ha incrementado por efecto del cambio en el clima (Cadima et al. 2016). El principal problema identificado es el tizón tardío (Phytophthora infestans), aunque los agricultores también mencionaron como importante al tizón temprano (Alternaria solani).

Determinación de rasgos funcionales importantes de la papa. Para efectos de la evaluación de la diversidad de papa de Colomi, se consideraron los siguientes atributos (variables) categorizados en rasgos funcionales:

- Medioambientales: bióticos (resistencia a tizón tardío y resistencia a tizón temprano) y abióticos (tolerancia a heladas, granizadas, humedad del suelo $\mathrm{y}$ sequía).

- Agronómicos: ciclo del cultivo, rango altitudinal de producción, rendimiento.
- Mercado: mercado actual, apto para procesamiento tradicional (tunta $\mathrm{y}$ chuño)

- Calidad: caracterización energética local, apreciación culinaria local, tiempo de cocción

Evaluación de la cantidad y distribución de la diversidad de rasgos funcionales en los cultivares de papa. Los 37 cultivares de papa (30 nativos y 7 mejorados) de Colomi fueron colectados de diferentes comunidades del Municipio y sembrados en una parcela para conformar el jardín de cultivares de papa de Colomi, donde se realizaron evaluaciones participativas y toma de datos con informantes clave. Cada cultivar contó con tres a cinco plantas en la parcela. Se registraron los nombres comunes, la especie y el origen (nativo o mejorado).

La resistencia a tizón tardío y tizón temprano se evaluó en cada planta de cada cultivar con criterios de los agricultores. Aunque no representa una evaluación exhaustiva con metodologías estándar como la determinación del área bajo la curva de progreso de la enfermedad (AUDPC por sus siglas en inglés) recomendadas por varios autores (Barquero et al. 2005; Gabriel et al. 2007; Pérez y Forbes 2008), se ajustaron los criterios de los agricultores a una escala práctica tomando en cuenta la siguiente puntuación: $0=$ susceptible ( $>50 \%$ daño del follaje); $1=$ moderadamente susceptible (20 a $50 \%$ daño); $2=$ resistente $(<20 \%$ daño $)$. Para cada cultivar se obtuvo el promedio de las plantas evaluadas.

La reacción a heladas se evaluó tomando datos del daño al follaje de cada planta en una escala de 1 a 9 sugerida por Mendoza y Estrada (1979), adecuado por Choque et al. (2007). Cuando el promedio del daño del efecto de la helada sobre las plantas fue $\geq 3.0$, entonces $0=$ cultivar susceptible; si el promedio fue $<3$, entonces $1=$ tolerante. $\mathrm{La}$ reacción a las granizadas $(0=$ susceptible, $1=$ moderadamente susceptible, $2=$ tolerante), la tolerancia a la humedad del suelo $(0=$ susceptible, $1=$ tolerante) y la tolerancia a la sequía $(0=$ susceptible, $1=$ tolerante $)$ se 
evaluaron tomando en cuenta el conocimiento local de los informantes clave.

El ciclo del cultivo se consideró en días desde la fecha de siembra hasta la cosecha de los tubérculos. La información fue complementada con los agricultores quienes identificaron diferentes ciclos para algunos de los cultivares de acuerdo al piso altitudinal donde es plantado, por lo que el ciclo de cultivo fue categorizado como: $0=\operatorname{precoz}(90$ a 120 días), 1 = semiprecoz/semitardío (130 a 140 días), 2 = tardío ( $\geq 150$ días), $3=$ cuando es precoz en zonas bajas y semitardío en zonas altas, $4=$ cuando es semitardío en zonas bajas y tardío en zonas altas. La referencia de precocidad se tomó de Gabriel et al. (2011).

El rango altitudinal de producción también fue determinado por los agricultores y se encontraron cinco categorías: $1=$ cabecera valle/puna baja (2.500 a $3.300 \mathrm{msnm}), 2=$ puna alta (3.900 a $4.200 \mathrm{msnm}), 3=$ puna baja a puna alta $(2500$ a $4200 \mathrm{msnm}), 4=$ ladera a puna alta $(3500$ a $4200 \mathrm{msnm}), 5=$ puna baja a ladera (2500 a $3700 \mathrm{msnm})$. El rendimiento fue calculado en base a los datos de evaluación de la parcela complementado con información de los agricultores, por ello se reportó el rendimiento en toneladas por hectárea ( $\mathrm{t} / \mathrm{ha}$ ) bajo buenas condiciones de cultivo (medio ambientales y de manejo) y bajo condiciones de estrés.

En base a los informantes clave se determinó para cada cultivar si tiene mercado actual o solo es producido para consumo familiar. También se determinó si los cultivares tenían aptitud de procesamiento en chuño tunta y chuño negro. El chuño es una forma de conservación a largo plazo, que consiste en el congelamiento del tubérculo seguido por una deshidratación. La caracterización energética local $(0=$ pisi (poco energético), $1=$ kallpalchador (energético)) tiene que ver con la harinosidad del tubérculo (más harinoso más energético). La apreciación culinaria local fue determinada en $0=$ malo, $1=$ regular, 2 = bueno, en base al gusto de la gente local. El tiempo de cocción fue determinado en laboratorio, con valores de 0
= lento (tiempo de cocción $>2 \mathrm{~min} / \mathrm{cm} 3 \mathrm{de}$ tubérculo), $1=$ medio $(1-2 \mathrm{~min} / \mathrm{cm} 3), 2=$ rápido $(<1 \mathrm{~min} / \mathrm{cm} 3)$.

Una vez tabulados todos los datos, éstos fueron validados/confirmados y/o ajustados con productores informantes clave de Colomi.

\section{Análisis de los datos e identificación de los repertorios de cultivares}

Debido a que los rasgos funcionales de los cultivares de papa estaban conformados por un conjunto de variables mixtas (cuantitativas y cualitativas), se utilizó un Análisis de Factores para Datos Mixtos (Pagès 2014) para a) determinar la asociación entre las variables, b) evaluar tendencias de agrupación entre los diferentes cultivares de papa en función a los rasgos funcionales, y finalmente, $c$ ) determinar la potencialidad de los cultivares de papa solo con las variables más atractivas para el cambio climático (tizón tardío, sequía, y rendimiento) pero sin dejar de lado la apreciabilidad culinaria. Todos los análisis estadísticos y gráficos fueron realizados en el lenguaje de libre distribución $\mathrm{R}$ v3.4.1, con los paquetes FactoMineR (Le et al. 2008) y factoextra (Kassambara y Mundt 2017), respectivamente.

\section{Resultados y discusión}

La Figura 1 muestra que los agricultores de Colomi mantienen y cultivan una diversidad importante de papa. Esta diversidad ya fue evidenciada en estudios hace 10 y 20 atrás (Terrazas y Valdivia 1998, Terrazas et al. 2007), y aún es posible encontrarla en la actualidad (Cadima et al. 2016). La diversidad de cultivares nativos es mayor la que la diversidad de los cultivares mejorados, y aparentemente éstos últimos no han desplazado a la diversidad nativa, más bien han sido adoptados en la producción familiar aunque la mayoría son producidos para el mercado. El análisis de este estudio se limita al número de cultivares encontrados en Colomi. Otros estudios han analizado el desplazamiento de la diversidad nativa en términos de superficie cultivada y han mostrado que al menos el $50 \%$ de los cultivares nativos están bajo riesgo de pérdida 
porque son pocos los agricultores que cultivan estos materiales en pequeñas superficies, y que más del $60 \%$ de los cultivares mejorados son cultivados por muchos agricultores, la mayoría en grandes superficies (Cadima 2016). El presente estudio evidencia además que las papas de Colomi, tanto cultivares nativos como mejorados, contienen importantes rasgos funcionales medioambientales, agronómicos, de mercado y de calidad, con una variación importante dentro de cada rasgo, lo cual es relevante para cubrir las necesidades actuales de los agricultores ante las adversidades climáticas y medio ambientales, como resistencia a las enfermedades y tolerancia a factores abióticos. La mayor parte de los cultivares son de ciclo tardío, lo cual es una desventaja ante periodos de lluvia reducidos por el cambio climático, pero una mayor amplitud en el rango altitudinal de producción podría constituirse en una ventaja para escapar a los problemas medioambientales. Llama la atención también que la mayoría de los cultivares no tienen mercado (55\% de los cultivares), sin embargo este hecho debería considerarse una oportunidad, ya que hay un gran número de cultivares que podrían ser atractivos para la producción y consumo a mayor escala, debido a su resistencia a varias causas de estrés y a su cualidades organolépticas.

El Análisis de Factores para Datos Mixtos evidenció que los rasgos funcionales relacionados con la resistencia al tizón tardío, el rango altitudinal y la tolerancia al granizo son las variables que mejor explican la mayor variabilidad de las papas de Colomi (Tabla 1). Sin embargo la variación acumulada sugiere tomar con cautela la interpretación del análisis porque solo cerca del $35 \%$ de toda la variabilidad entre los cultivares analizados se concentra entre las dimensiones (ejes) 1 al 3, y recién se llega al 50\% con cinco dimensiones (Tabla 2).

Tabla 1. Correlación de las variables explicativas con cada eje o dimensión

\begin{tabular}{lccccc|lccccc}
\hline Variable & Dim 1 & Dim 2 & Dim 3 & Dim 4 & Dim 5 & Variable & Dim 1 & Dim 2 & Dim 3 & Dim 4 & Dim 5 \\
\hline RendB & 0,0848 & 0,2342 & 0,0140 & 0,0056 & 0,1272 & Sequía & 0,1187 & 0,0054 & 0,0436 & 0,1794 & 0,0340 \\
RendS & 0,5021 & 0,1212 & 0,0050 & 0,0223 & 0,0003 & Tunta & 0,1252 & 0,1257 & 0,0401 & 0,4223 & 0,0169 \\
Origen & 0,3834 & 0,1322 & 0,0366 & 0,0004 & 0,0181 & Chuño & 0,1483 & 0,0015 & 0,0102 & 0,0372 & 0,2330 \\
TizónTa & $\mathbf{0 , 7 0 2 2}$ & 0,0952 & 0,1741 & 0,0911 & 0,0159 & Energía & 0,1582 & 0,0744 & 0,0142 & 0,0590 & 0,0648 \\
TizónTe & 0,1583 & 0,1953 & 0,0708 & 0,3677 & 0,0028 & Ciclo & 0,2828 & 0,4198 & 0,0551 & 0,2886 & 0,1622 \\
Helada & 0,0162 & 0,0726 & 0,2578 & 0,1910 & 0,1835 & Aprecia & 0,0400 & 0,2395 & 0,4549 & 0,0757 & 0,1323 \\
Granizo & $\mathbf{0 , 5 1 2 6}$ & 0,3830 & 0,2375 & 0,0441 & 0,3007 & Mercado & 0,1164 & 0,2705 & 0,0004 & 0,1698 & 0,0583 \\
Humedad & 0,1345 & 0,0831 & 0,3151 & 0,0223 & 0,0923 & Altitud & 0,4766 & 0,1749 & $\mathbf{0 , 6 5 5 7}$ & 0,1139 & 0,4496 \\
\hline
\end{tabular}

Donde: RendB=rendimiento bajo buenas condiciones de cultivo, RendS=rendimiento bajo condiciones de estrés, TizónTa=tizón tardío, TizónTe=tizón temprano

Tabla 2. Valores propios (eigenvalues) y variación acumulada

\begin{tabular}{|c|c|c|c|c|c|c|}
\hline \multirow{2}{*}{ Dimensiones* } & \multicolumn{3}{|c|}{ Valores con todas la variables } & \multicolumn{3}{|c|}{$\begin{array}{c}\text { Valores con variables más críticas para el cambio } \\
\text { climático }\end{array}$} \\
\hline & eigenvalue & $\begin{array}{l}\text { Variación } \\
\%\end{array}$ & $\underset{\%}{\text { Acumulado }}$ & eigenvalue & $\begin{array}{l}\text { Variación } \\
\%\end{array}$ & $\underset{\%}{\operatorname{Acumulado}}$ \\
\hline Dim 1 & 3.960281 & 15.231852 & 15.23185 & 1.4391372 & 23.98562 & 23.98562 \\
\hline Dim 2 & 2.628309 & 10.108881 & 25.34073 & 1.3054436 & 21.75739 & 45.74301 \\
\hline Dim 3 & 2.385007 & 9.173103 & 34.51384 & 1.0883346 & 18.13891 & 63.88192 \\
\hline Dim 4 & 2.090407 & 8.040025 & 42.55386 & 0.9269772 & 15.44962 & 79.33154 \\
\hline Dim 5 & 1.891728 & 7.275878 & 49.82974 & 0.7390258 & 12.31710 & 91.64864 \\
\hline
\end{tabular}

*el paquete FactoMineR utiliza por defecto cinco dimensiones para visualizar la proporción de varianzas retenidas por las diferentes dimensiones (Pagès, 2014) 
El análisis estadístico ayudó aunque limitadamente a definir tendencias de agrupación de cultivares en función a los rasgos funcionales. La gráfica con las dimensiones 1 y 2 (Figura 2A) permite visualizar un grupo mixto de cultivares nativos y mejorados con resistencia a tizón tardío, con ciclo de cultivo semiprecoz y cultivados en ambientes desde la puna baja a ladera (Sani Imilla, Papa phureja, Pali, Yuca papa, Puca waycha, Robusta y Qhori sunqu), grupo que se confirma en la gráfica con las dimensiones 1 y 3 (Figura 2B). Un segundo grupo, aunque menos evidente, puede visualizarse en la Figura 2B de solo cultivares nativos, éstos tienden a ser susceptibles a tizón tardío, pero se caracterizan por ser altamente apreciados por su calidad culinaria y porque tienen una buena amplitud de siembra desde la puna baja a alta, lo cual podría ser una ventaja para reducir los riesgos abióticos al sembrar en diferentes zonas de cultivo (Yuraj canastillo, Runa papa, Puca qoyllu, Yana qoyllu, Tanta wawa, Machu wañichu, Pitu wawa, Kellu sunqu, Yana runa y Katawi). Aunque no existen estudios oficiales sobre la plasticidad de los cultivares de papa nativa en cuanto a la altitud, el conocimiento local de los agricultores en este estudio ha categorizado la aptitud de los cultivares para adaptarse a diferentes zonas altitudinales. De hecho, los cultivares mejorados no se adaptan a zonas muy altas para producción comercial, llegan máximo hasta la ladera $(3700 \mathrm{~m})$, aunque los agricultores llevan todos los cultivares a las zonas altas para producir o refrescar la sanidad de la semilla, una práctica ancestralmente utilizada en los Andes (Thiele 1999).

El análisis reduciendo las variables a las más atractivas para el cambio climático, mostró una variación acumulada de cerca del $64 \%$ con las tres primeras dimensiones (Tabla 2), y la tendencia de agrupación de cultivares mostró un grupo resistente al tizón tardío similar al anterior análisis (Papa Phureja, Yuca papa, Robusta, Puca Waycha, Quri sunqu) (Figura 3C), que se confirma en la Figura 3D. Otros dos posibles grupos, aunque son menos evidentes, podrían extraerse de la Figura 3D, uno con los cultivares que son altamente apreciados por sus características culinarias pero que aparentemente no tendrían cualidades para enfrentar el cambio climático (Puca qoyllu, Yana qoyllu, Candelero, Yana Runa), y otro posible grupo con los cultivares que son tolerantes a la sequía, medianamente susceptibles a tizón tardío y apreciados por sus características culinarias (Pinta boca, Chejchi canastillo, Puca ñawi, Waca qallu, Runa Toralapa).

Los datos evidenciaron que los cultivares que en la actualidad tienen mercado presentan algunos atributos en común como que tienen altos rendimientos (16 a $\geq 20 \mathrm{t} / \mathrm{ha}$ ), una buena a alta apreciación culinaria local, tolerancia a sequía y resistencia a tizón tardío (moderado a resistente). Coincidentemente estas mismas variables fueron elegidas en el estudio como las más atractivas para el cambio climático. Aunque en el análisis estadístico no se incluyó la variable de mercado, los grupos de cultivares obtenidos incluyeron cultivares que no tienen mercado actual pero tienen potencial de rendimiento.

Los criterios que se proponen con los resultados de este trabajo para seleccionar cultivares en el contexto del cambio climático son: además de los anteriormente citados (buena a alta apreciación culinaria local, tolerancia a sequía y resistencia a tizón tardío), incluir el ciclo del cultivo (precocidad y semiprecocidad) y la amplitud altitudinal, estos últimos rasgos como medidas de escape a problemas medioambientales.

En este trabajo los productores definieron sus necesidades y preferencias según las condiciones ambientales, preferencias agronómicas, de mercado y valores culturales que son importantes para ellos. Los rasgos funcionales se identificaron conforme a estas necesidades y preferencias definidas por los propios agricultores. La definición de repertorios de cultivares podría eventualmente ser diferente si se consideraran las necesidades y preferencias de otros actores, como consumidores externos y la industria. 
Las necesidades y preferencias fueron priorizadas en base a las especificidades del lugar de evaluación, en este caso a las condiciones de Colomi (clima, topografía, suelos). La priorización de las necesidades y preferencias podrían también ser diferentes al considerar prioritarios otros factores como el destino y los usos de los cultivares (ejemplo mercado).

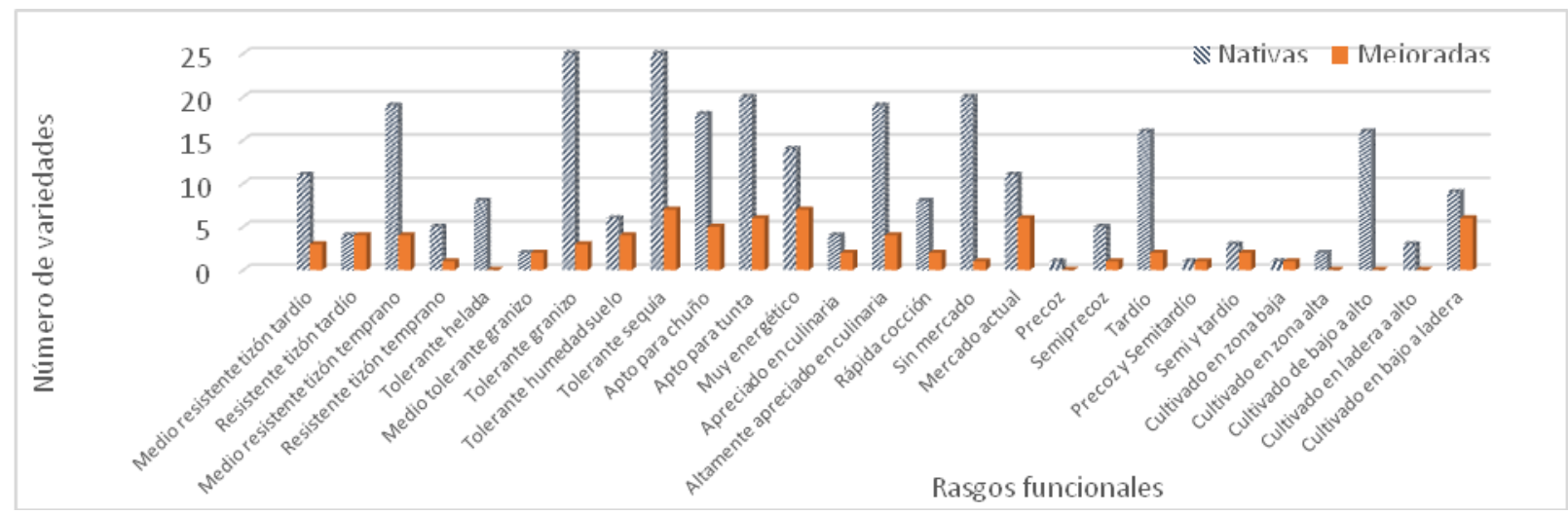

Figura 1. Número de cultivares de papa nativas y mejoradas cultivadas en Colomi que manifiestan un rango de diferentes rasgos funcionales del tipo medioambiental, agronómico, de mercado y calidad.

Un segundo grupo, aunque menos evidente, puede visualizarse en la Figura $2 \mathrm{~B}$ de solo cultivares nativos, éstos tienden a ser susceptibles a tizón tardío, pero se caracterizan por ser altamente apreciados por su calidad culinaria y porque tienen una buena amplitud de siembra desde la puna baja a alta, lo cual podría ser una ventaja para reducir los riesgos abióticos al sembrar en diferentes zonas de cultivo (Yuraj canastillo, Runa papa, Puca qoyllu, Yana qoyllu, Tanta wawa, Machu wañichu, Pitu wawa, Kellu sunqu, Yana runa y Katawi). Aunque no existen estudios oficiales sobre la plasticidad de los cultivares de papa nativa en cuanto a la altitud, el conocimiento local de los agricultores en este estudio ha categorizado la aptitud de los cultivares para adaptarse a diferentes zonas altitudinales. De hecho, los cultivares mejorados no se adaptan a zonas muy altas para producción comercial, llegan máximo hasta la ladera $(3700 \mathrm{~m})$, aunque los agricultores llevan todos los cultivares a las zonas altas para producir o refrescar la

Los datos duros evidenciaron que los cultivares que en la actualidad tienen mercado, presentan algunos atributos en sanidad de la semilla, una práctica ancestralmente utilizada en los Andes (Thiele 1999).

El análisis reduciendo las variables a las más atractivas para el cambio climático, mostró una variación acumulada de cerca del $64 \%$ con los tres primeros componentes (Tabla 2), y la tendencia de agrupación de cultivares mostró un grupo resistente al tizón tardío similar al anterior análisis (Papa Phureja, Yuca papa, Robusta, Puca Waycha, Quri sunqu) (Figura 3C), que se confirma en la Figura 3D. Otros dos posibles grupos, aunque son menos evidentes, podrían extraerse de la Figura 3D, uno con los cultivares que son altamente apreciados en la culinaria pero aparentemente no tendrían cualidades para enfrentar el cambio climático (Puca qoyllu, Yana qoyllu, Candelero, Yana Runa), y otro posible grupo con los cultivares que son tolerantes a la sequía, mediamente susceptibles a tizón tardío y apreciados en la culinaria (Pinta boca, Chejchi canastillo, Puca ñawi, Waca qallu, Runa Toralapa).

común como que tienen altos rendimientos (16 a $\geq 20 \mathrm{t} / \mathrm{ha}$ ), una buena a alta apreciación culinaria local, tolerancia a sequía $\mathrm{y}$ 
resistencia a tizón tardío (moderado a resistente). Coincidentemente estas mismas variables fueron elegidas en el estudio como las más atractivas para el cambio climático, aunque en el análisis estadístico no se incluyó la variable de mercado, los grupos de cultivares obtenidos incluyeron cultivares que no tienen mercado actual pero tienen potencial de rendimiento.

Los criterios que se proponen con los resultados de este trabajo para seleccionar cultivares en el contexto del cambio climático son: además de los anteriormente citados, incluir el ciclo del cultivo (precocidad y semiprecocidad) y la amplitud altitudinal, estos últimos rasgos como medidas de escape a problemas medioambientales.

En este trabajo se definieron necesidades y preferencias de los productores para identificar los rasgos funcionales que respondan a esas necesidades y preferencias asociadas a condiciones medioambientales, preferencias agronómicas, de mercado y valores culturales. La definición de portafolios de cultivares podría eventualmente ser diferente si se consideraran las necesidades y preferencias de otros actores, como consumidores externos y la industria.

Las necesidades y preferencias fueron priorizadas en base a las especificidades del lugar de evaluación, en este caso a las condiciones de Colomi (clima, topografía, suelos). La priorización de las necesidades y preferencias podrían también ser diferentes al considerar prioritarios otros factores como el destino y los usos de los cultivares (ejemplo mercado).

\section{Conflictos de intereses}

Los autores no reportan conflicto de interés en el desarrollo de este estudio.

\section{Agradecimientos}

Se agradece el apoyo financiero de Bioversity International, y a Devra Jarvis, por su consejo y guía general a la hora de definir y aplicar el método para identificar repertorios de cultivares según rasgos funcionales, también la colaboración de Alder Keleman de Yale
University, Rhimer Gonzales y Gonzalo Tiñiñi de PROINPA en la colecta de datos, y Melina Campero de la Universidad Mayor de San Simón para el análisis estadístico.

\section{Referencias citadas}

Barquero, M.; Gómez, L.; Brenes, A. 2005. Resistencia al tizón tardío (Phytophthora infestans) en clones promisorios de papa en Costa Rica. Agronomía Costarricense 29(3): $31-45$

Cadima, X.; Gonzales, R.; Delfín, M.; Patiño, F. (2016). La agrobiodiversidad de Colomi en un contexto de cambio climático. Fundación PROINPA, Cochabamba, Bolivia. 108 p.

Cadima, X. (2016). Final technical report Bolivia Project "Improving the availability and use of diverse seed and other planting materials to reduce vulnerability and improve food security for smallholders in vulnerable ecosystems". Fundación PROINPA Bioversity International. Cochabamba, Bolivia.

Cadima, X.; Gandarillas, A. (2009). Conserving Bolivia's potatoes. pp. 20. En: Geneflow 2009. Bioversity International. Rome, Italy. 64 p.

Choque, E.; Espinoza, R.; Cadima, X.; Zeballos, J.; Gabriel J. (2007). Resistencia a helada en germoplasma de papa nativa de Bolivia. Revista Latinoamericana de la Papa 14 (1): 24-32.

Escobar, A.G. (2015). Effects of National Policies and Value Chain in potato seed diversity in the Municipality of Colomi (Cochabamba-Bolivia). School of Agricultural, Forest and Food Sciences, Bern University of Applied Sciences, Swizterland. $93 \mathrm{p}$.

Eyzaguirre, J.L. (2015). Importancia socioeconómica de la agricultura familiar en Bolivia. TIERRA, La Paz, Bolivia. 92 p.

García, W.; Cadima X. (eds). (2003). Manejo sostenible de la agrobiodiversidad de tubérculos andinos: Síntesis de investigaciones y experiencias en Bolivia. Conservación y uso de la biodiversidad de raíces y tubérculos andinos: Una década de 
investigación para el desarrollo (1993-2003). 1. Fundación PROINPA, Alcaldía de Colomi, CIP, COSUDE. Cochabamba, Bolivia. 208 p.

Gabriel, J.; Forqueda, F.; Plata, G.; Fernández-Northcote, E. (2007). Caracterización de genotipos de papa de Europa y Latinoamérica por resistencia a tizón y propiedades culinarias. Revista Latinoamericana de la Papa 14(1): 10-23.

Gabriel, J.; Pereira, R.; Gandarillas, A. (2011). Catálogo de nuevas variedades de papa en Bolivia. Fundación PROINPA, Cochabamba, Bolivia. 55 p.

Gobierno Autónomo Municipal (GAM) Colomi. (2009). Ajuste y Complementación del Plan de Desarrollo Municipal de Colomi 2009-2013. Instituto de Investigaciones de Arquitectura - UMSS. Cochabamba (Bolivia). 200 p.

INE. (2018). Instituto Nacional de Estadística. Superficie cultivada por año agrícol según cultivos. Periodo 1983 -2013. En:

https://www.ine.gob.bo/index.php/estadistica s-por-actividad-economica/. Consulta: Abril, 2018.

Kassambara, A.; Mundt, F. (2017). Factoextra: Extract and Visualize the Results of Multivariate Data Analyses. R package version 1.0.4. En: https://CRAN.R- project.org/package=factoextra..$\quad$ Consulta: Julio, 2017.

Le, S. ; Josse, J.; Husson, F. (2008). FactoMineR: An R Package for Multivariate Analysis. Journal of Statistical Software 25(1): 1-18.

Pagès, J. (2014). Multiple Factor Analyses by example using R. CRC Press, Taylor \& Francis Group; Florida, USA. 272 p.

Pérez, W., Forbes, G. (2008). Manual Técnico. El tizón tardío de la papa. Centro Internacional de la Papa, Lima, Perú. 39 p.

Terrazas, F.; Valdivia, G. (1998). Space Dynamics of in situ Preservation: Handling of the Genetic Diversity of Andean Tubers in Mosaic Systems Candelaria, Cochabamba (Bolivia). En: Plant Genetic Resources Newsletter 114:9-15.

Terrazas, A.F.; Baudoin, J.P.; Gonzalo, D.P. (2007). Procesos dinámicos locales para la conservación in situ de la diversidad genética de tubérculos andinos cultivados en el microcentro de Candelaria (Cochabamba, Bolivia). En: Plant Genetic Resources Newsletter 152: 1-11.

Thiele, G. (1999). Informal potato seed systems in the Andes: Why are they important and what should we do with them?. World Development 27:83-99. 

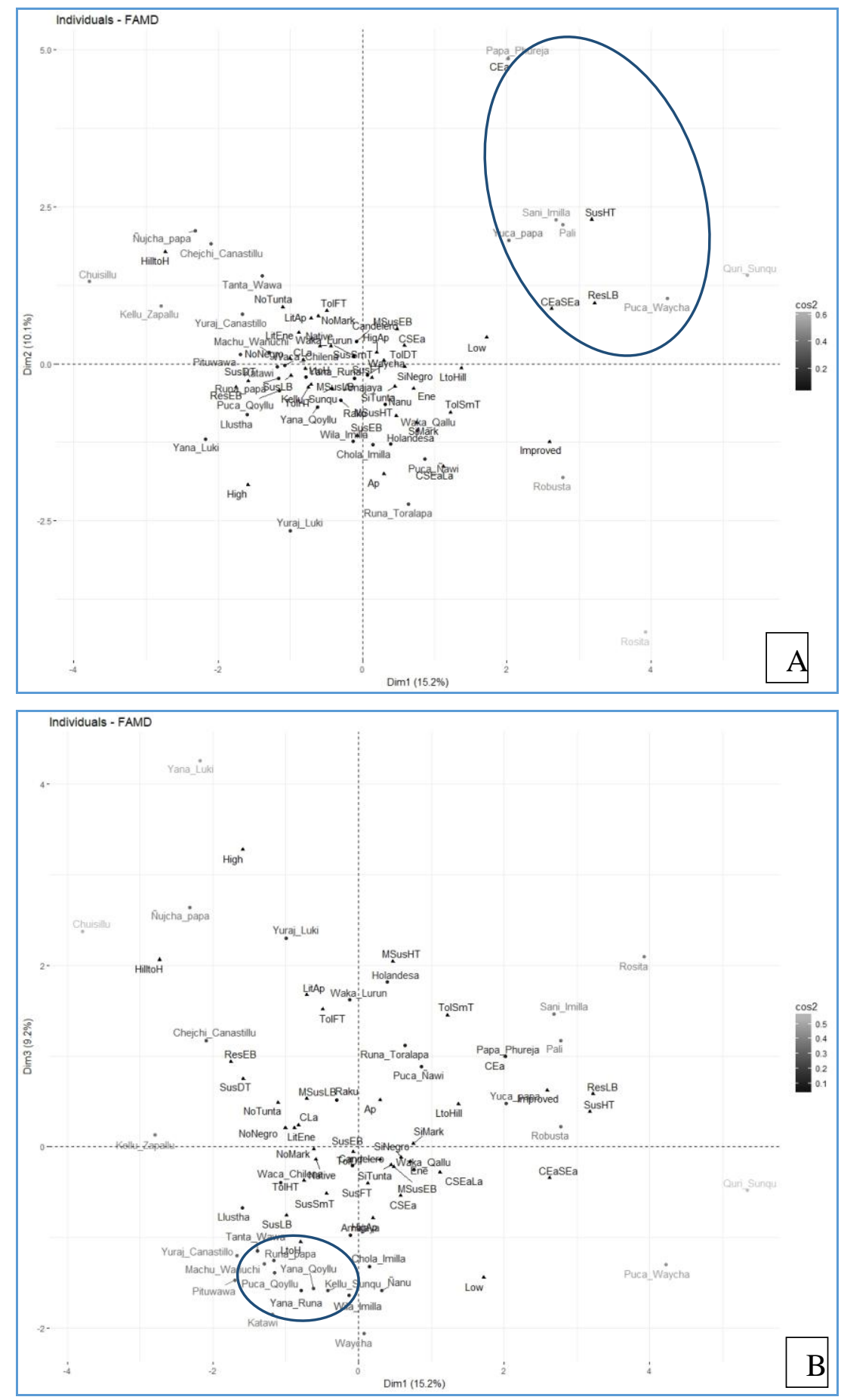

Figura 2. Agrupación de cultivares de papa de Colomi, A) y B) considerando todas las variables. A) con las dimensiones 1 y 2, B) con las dimensiones 1 y 3 . 

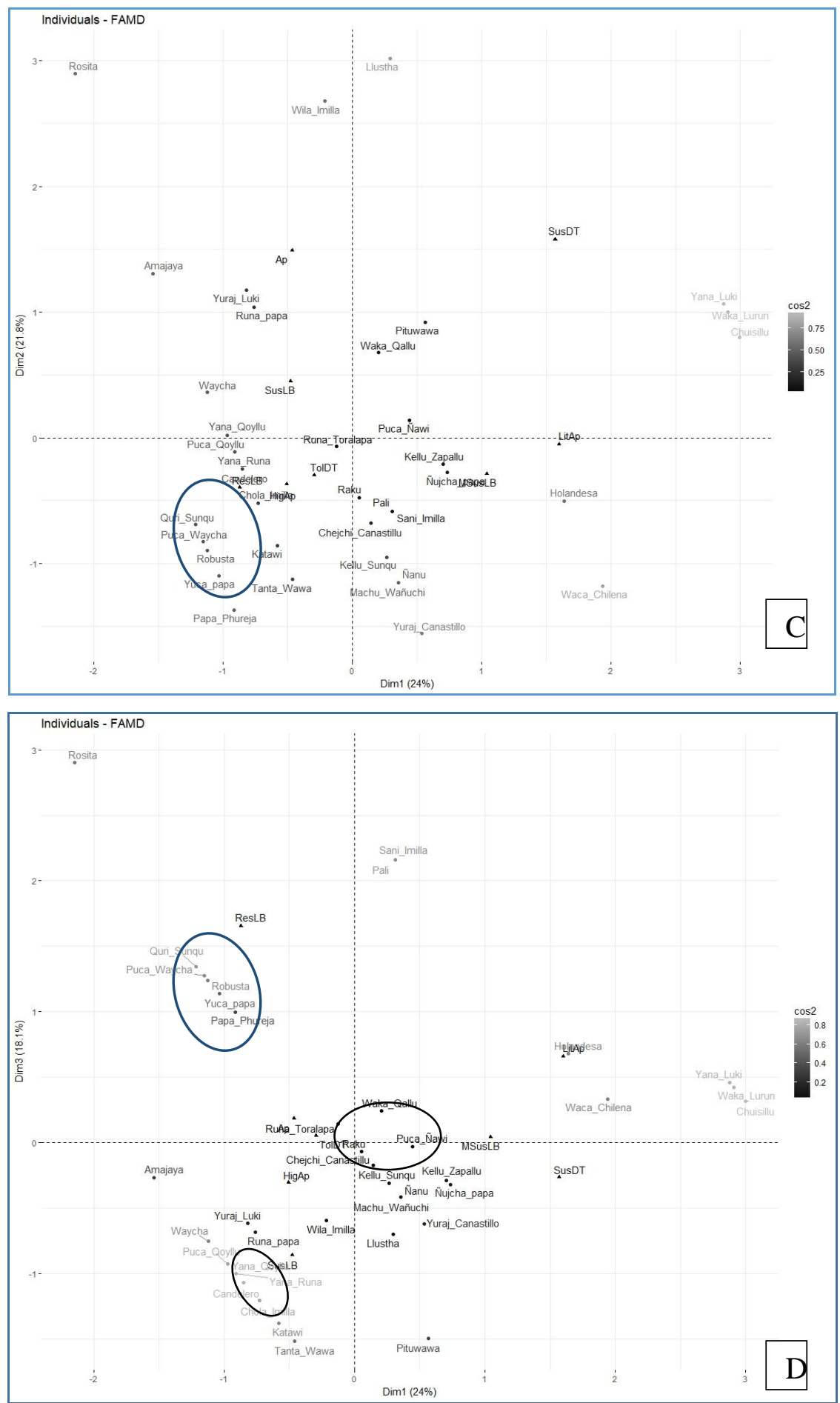

Figura 3. Agrupación de cultivares de papa de Colomi, C) y D) considerando solo variables para el cambio climático. C) con las dimensiones 1 y 2, D) con las dimensiones 1 y 3. 